\title{
Coherent Oscillations of Turbulent Rayleigh-Bénard Convection in a Thin Vertical Disk
}

\author{
Hao Song, ${ }^{1}$ E. Villermaux, ${ }^{2}$ and Penger Tong ${ }^{1}$ \\ ${ }^{1}$ Department of Physics, Hong Kong University of Science and Technology, Clear Water Bay, Kowloon, Hong Kong \\ ${ }^{2}$ Université de Provence, IRPHE, 13384 Marseille Cedex 13, France
}

(Received 24 January 2011; published 6 May 2011)

\begin{abstract}
A well-defined oscillation is observed in the power spectrum of several fluctuating signals in turbulent Rayleigh-Bénard convection occurring in a thin vertical disk filled with water. The experiment reveals that the coherent oscillations are produced by periodic emission of thermal plumes, which gives rise to periodic pulses of forcing, resulting in a pulsed large-scale circulation in the thin cell. The experimental results agree well with the theoretical predictions made from two coupled nonlinear delayed equations.
\end{abstract}

Turbulent Rayleigh-Bénard convection, where a fluid layer of thickness $H$ is heated from below and cooled from the top, has served as a model system for the study of a range of interesting problems in fluid dynamics [1,2]. One of the intriguing features of turbulent convection is the emergence of a well-defined oscillation frequency in the temperature [3,4] and velocity [5] power spectra at large Rayleigh numbers. The oscillations in the temperature and velocity fields are synchronized [6] and are associated with the dynamics of the large-scale circulation (LSC) [7-9]. Despite the large number of experimental studies [3-10], the origin of the coherent oscillations has eluded a number of attempts.

(i) Howard [11] first proposed that the thermal boundary layer near a conducting plate grows in time and becomes unstable when its thickness $\delta$ becomes larger than a critical value. Such a boundary layer instability produces periodic emission of thermal plumes at a diffusion-limited rate, $f_{0} \sim \kappa / \delta^{2}$, where $\kappa$ is the thermal diffusivity of the convecting fluid. (ii) In 1995, Villermaux [12] proposed a deterministic model, in that the boundary layer instability is triggered by the incoming thermal plumes transported along the cell periphery by LSC. As a result, the thermal plumes are excited alternately between the boundary layers with a local frequency $f_{0} \simeq 1 / \tau_{t}$, where $\tau_{t} \simeq 2 H / U$ is the turnover time of LSC with $U$ being the velocity of LSC. (iii) Recently, Brown and Ahlers [13] proposed two coupled stochastic equations to describe the motion of LSC. Under certain assumptions, the governing equation becomes that for an underdamped oscillator with a restoring force produced by a wall-generated pressure gradient, when LSC deviates from its preferred orientation. The resonant frequency around the local potential minimum is $f_{0} \sim U / H$. (iv) The last model, which was proposed by Araujo et al. [14], considered a scenario in which insufficient heat exchange occurs because of temporal or spatial inhomogeneities in turbulent convection. Such inhomogeneities may cause accumulations of heat over time or at certain locations (hot spots), which can produce reversals or oscillations of LSC at a frequency $f_{0} \simeq \kappa / \ell^{2}$, where $\ell$ is a characteristic length at which the local inhomogeneities occur.

Up to now, most of the convection experiments aimed at testing the above theoretical models were conducted in upright cylindrical cells with an aspect ratio close to unity [3-10]. Our current understanding of the structure and dynamics of LSC is also built upon this body of experimental results. A natural question regarding this system is, to what extent can the obtained experimental results be applied to other convection systems? To answer this question, one needs to change either the aspect ratio or shape of the convection cell and test the theory in different types of convection cells.

In this Letter, we report a systematic study of the coherent oscillations in a convection cell with different geometry. As shown in Fig. 1, the new cell has the shape of a thin disk with its circular plane orientated vertically parallel to gravity. The top and bottom $1 / 3$ of the circular sidewall are made of copper. Sandwiched in the middle of the sidewall

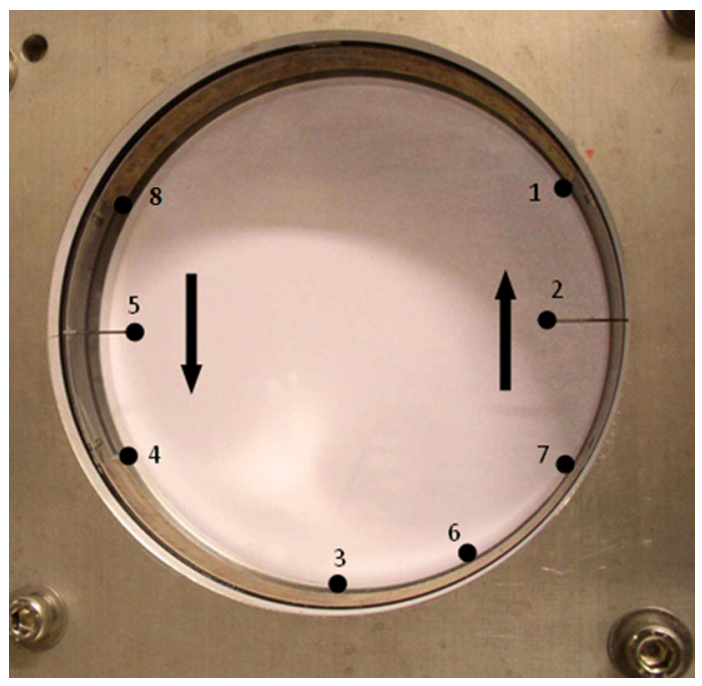

FIG. 1 (color online). End view of the thin convection cell. The numbers marked in the figure indicate the positions of local measurement. 
are two pieces of thermal insulating (curved) plate made of transparent Plexiglas. The two flat end walls of the cell are also made of the same Plexiglas. The cell has a circular cross section of inner diameter $D=18.8 \mathrm{~cm}$, and its thickness is $L=3.0 \mathrm{~cm}$. The corresponding aspect ratio is $\Gamma=L / D=0.16$. This cell is designed to simplify the large-scale flow. It has a circular cross section without any corner in order to prevent secondary flows, which can destabilize LSC [14]. Because the flow is confined in a thin circular disk, other flow modes such as torsional and sloshing oscillations $[9,13]$ cannot be excited in this quasi2D system. These simplifications allow us to have a stringent test of the theory.

The thin cell is filled with water and is placed inside a thermostat box, whose temperature matches the mean temperature of the bulk fluid. The Rayleigh number is defined as $\mathrm{Ra}=\alpha g \Delta T D^{3} /(\nu \kappa)$, where $g$ is the gravitational acceleration, $\Delta T$ is the temperature difference across the cell height (diameter) $D$, and $\alpha, \nu$, and $\kappa$ are, respectively, the thermal expansion coefficient, kinematic viscosity, and thermal diffusivity of the convecting fluid. In the experiment, the value of $\mathrm{Ra}$ is varied in the range $10^{8} \leqq \mathrm{Ra} \lesssim$ $10^{10}$, and the Prandtl number is fixed at $\operatorname{Pr}=\nu / \kappa \simeq 5.4$. The local temperature of the convecting fluid is measured by using a small waterproof thermistor and is sampled at $20 \mathrm{~Hz}$. Local velocity measurements are conducted by using a laser Doppler velocimetry system. A standard shadowgraph method is used to visualize the large-scale flow in the cell. Other details about the experiment have been described elsewhere [15].

Figure 2 shows the large-scale flow and the spatial distribution of thermal plumes in the thin cell. It is found that the thermal plumes organize themselves with rising warm plumes (darker) on the right and falling cold plumes (brighter) on the left, resulting in a large-scale circulation in the circular plane of the cell [16]. The fly-wheel-like flow structure is also observed in the measured velocity profile $U(r)$ along the radial direction [17]. The measured velocity has a zero mean at the cell center and increases linearly with the radial distance in the bulk region of the flow. Furthermore, we find that the measured Nusselt number (normalized heat flux) in the cell can be well described by a power law: $\mathrm{Nu}=0.255 \mathrm{Ra}^{0.28}$ [17]. At position 3 (see Fig. 1), we measure the maximum horizontal velocity $U_{3}$ of LSC with varying Ra. The corresponding Reynolds number $\operatorname{Re}=U_{3} D / \nu$ is also found to have a power law form: $\mathrm{Re}=0.042 \mathrm{Ra}^{0.49}$ [17]. These results reveal that this system possesses the key features of turbulent convection, which have been observed in the $\Gamma=1$ upright cylindrical cells.

Even in this confined space, where it is impossible to excite the torsional and sloshing flow modes $[9,13]$ in the horizontal directions, we observe a well-defined oscillation frequency in the power spectrum of five different signals. Figure 3 shows the obtained oscillation frequencies $f_{0}$ as a

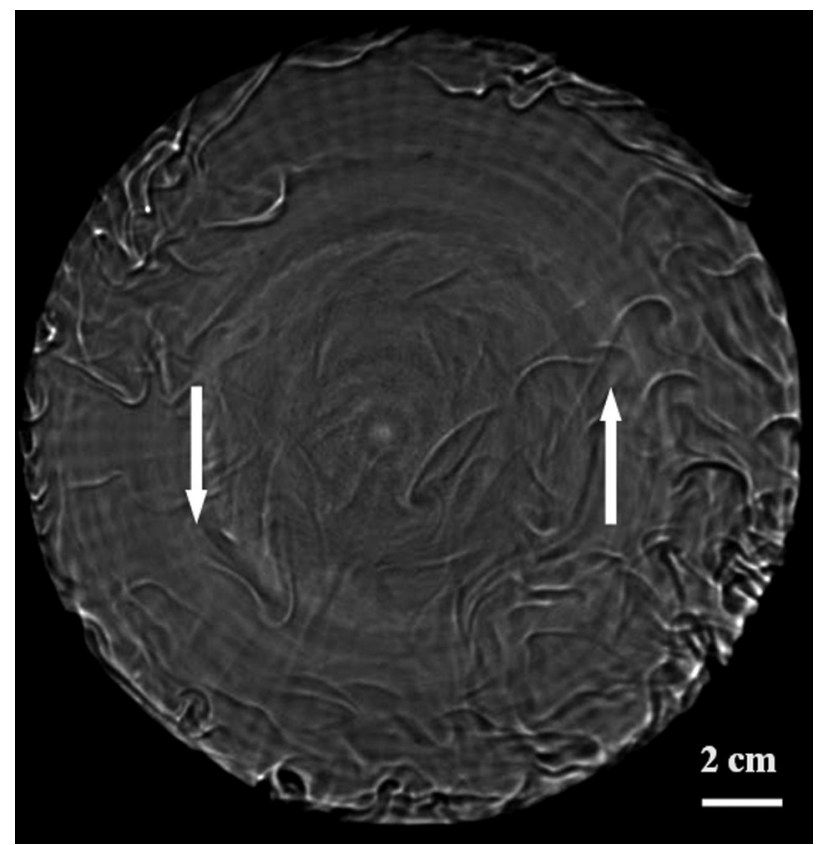

FIG. 2. A shadowgraph showing the large-scale flow and the spatial distribution of thermal plumes at $\mathrm{Ra}=4 \times 10^{9}$. The arrows indicate the direction of the large-scale flow.

function of Ra. In the plot, $f_{0}$ is normalized by $\tau_{0}=$ $\pi D /\left(4 U_{3}\right)$, which is one-quarter of the LSC turnover time. The open squares are obtained from the local (fluid) temperature at position 2. Identical oscillation frequencies are also obtained at other positions. The open circles are obtained from the temperature signals of a thermistor embedded in the bottom conducting plate near position 4 , which is very close to the edge of the conducting plate in

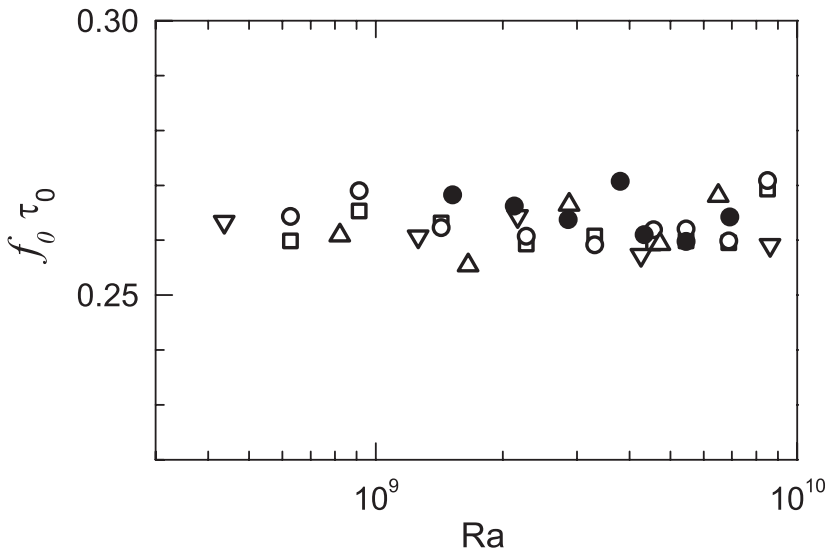

FIG. 3. Normalized oscillation frequencies $f_{0} \tau_{0}$ as a function of Ra. The oscillation frequencies are obtained from five different signals: (i) local (fluid) temperature at position 2 (open squares), (ii) local conducting plate temperature at position 4 (open circles), (iii) local horizontal velocity at position 3 (upward triangles), (vi) time-varying total heat flux of the top conducting plate (downward triangles), and (v) image intensity variation at position 4 (solid circles). 
contact with the insulating sidewall. The upward triangles are obtained from the measured $U_{3}(t)$ at position 3. The downward triangles are obtained from the time-varying total heat flux signals. The instantaneous heat flux is measured by using a thin film heat flux sensor (Captec), which covers the entire surface of the top copper plate with good thermal contact. Finally, the solid circles are obtained from the rms intensity variation $\delta I(t) \equiv \sum_{i}\left[I_{i}(t)-I_{0}(t)\right]^{2} / I_{0}(t)$ of the shadowgraph images sampled over a small area of $2 \times 3 \mathrm{~cm}^{2}$ at position 4 . Here $I_{0}(t)$ is the instantaneous mean value of the image intensity in the sampled area, and $\delta I(t)$ is defined in such a way that both the cold and warm plumes contribute to $\delta I(t)$. It is found that $\delta I(t)$ increases with the total number of thermal plumes in the sampled area [17]. Similar oscillations in $\delta I(t)$ are also found at position 1.

Figure 4 shows the typical power spectrum of four different fluctuating signals at $\mathrm{Ra}=3.3 \times 10^{9}$. All the power spectra reveal a common peak at $f_{0} \simeq 0.02 \mathrm{~Hz}$. A weaker second harmonic peak at $2 f_{0}$ is also observed for $\delta I(t)$ and local temperature signals. We find that the second peak is more sensitive to the noise and can be overwhelmed by turbulent noise when it is strong.

Figures 3 and 4 reveal several important features of the coherent oscillations in the thin cell. (i) All the oscillation frequencies collapse onto a single curve once they are normalized by $\tau_{t}$ (or $\tau_{0}$ ), suggesting that the oscillations are synchronized and are driven by the same mechanism. (ii) The value of $f_{0}$ is approximately equal to $1 / \tau_{t}$ for all values of $\mathrm{Ra}$, suggesting that $f_{0}$ has the same Ra dependence, $f_{0} \sim \mathrm{Ra}^{0.49}$, as $1 / \tau_{t}$ does. (iii) The observed oscillations in the total heat flux and in the corner region of the

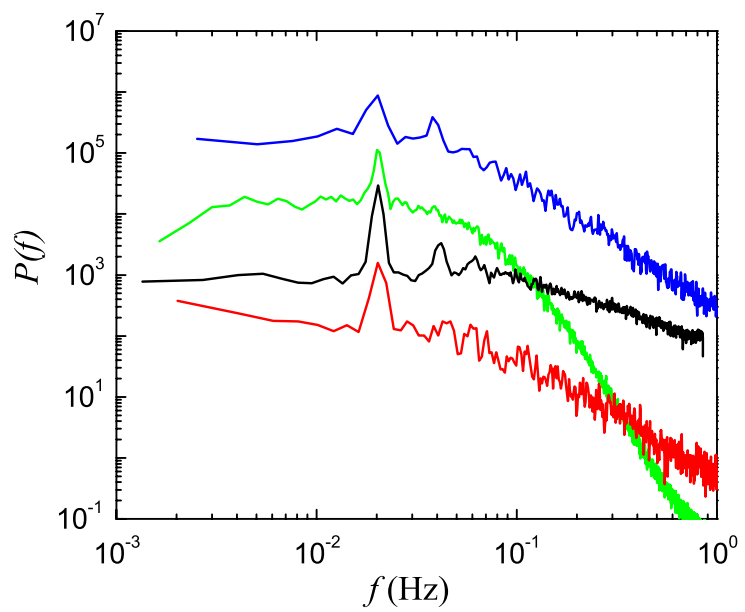

FIG. 4 (color online). Measured power spectrum of four different fluctuating signals at $\mathrm{Ra}=3.3 \times 10^{9}$ (from top to bottom): (i) image intensity variation at position 4 , (ii) instantaneous total heat flux of the top conducting plate, (iii) local (fluid) temperature at position 2, and (iv) local horizontal velocity at position 3 . For clarity, the amplitude of each power spectrum has been shifted slightly. conducting plate suggest that the thermal boundary layer is involved in the oscillation dynamics. (vi) The oscillations found for $\delta I(t)$ at positions 1 and 4 (or 7 and 8) suggest that the coherent oscillation is directly connected to periodic emission of cold and warm plumes in the opposing corner regions of the conducting plates.

From these features we conclude that the coherent oscillations are generated by the periodic emission of thermal plumes from the upper and lower thermal boundary layers, which produces a pulsed LSC in the thin cell. The experimental results discussed so far can be fully accounted by Villermaux's model [12], which explicitly involved interactions between the upper and lower boundary layers. To further test the theory, we examine the phase relation between the oscillating signals at different locations. In the experiment, we measure the cross-correlation function $C(\tau)=\left\langle A_{i}(t) A_{j}(t+\tau)\right\rangle /\left(\sigma_{i} \sigma_{j}\right)$ of the oscillating signal $A(t)$ between the positions $i$ and $j$ with $\sigma_{i}$ being the standard deviation at position $i$.

Figure 5 shows the measured $C(\tau)$ for temperature fluctuations between positions 2 and 5 (red curve) and for image intensity variations between positions 7 and 8 (blue curve). In both cases, the measured $C(\tau)$ shows a well-defined oscillation of frequency $f_{0}$. The image intensity variations at the two locations are in phase (blue curve), indicating that the two signals have no time delay. The temperature fluctuations at positions 2 and 5 are out of phase (red curve), because warm fluctuations at position 2 and cold fluctuations at position 5 have a sign difference. When the sign difference is taken into account, one finds that the temperature bursting at the two locations occurs at the same time.

To understand this fact, we reexamine the model in Ref. [12] consisting of two coupled nonlinear delayed

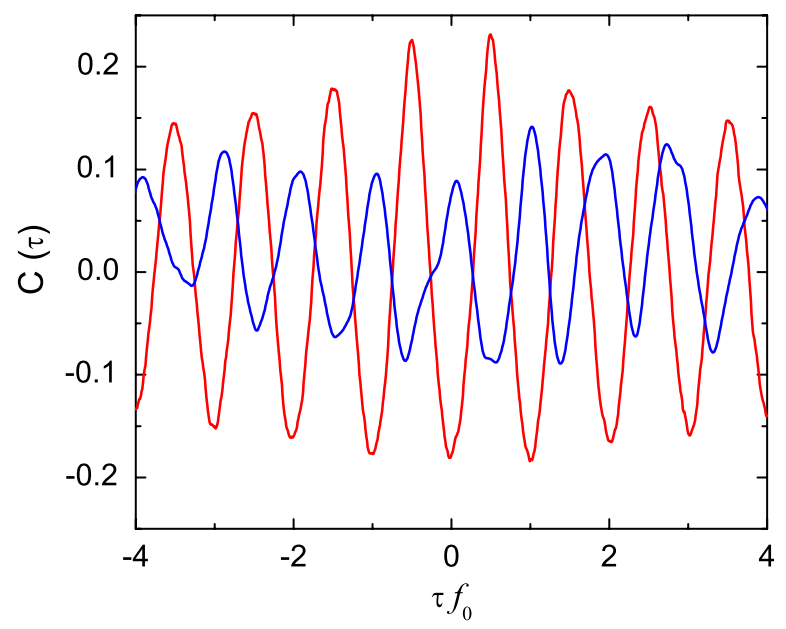

FIG. 5 (color online). Measured cross-correlation functions $C(\tau)$ as a function of the normalized delay time $\tau f_{0}$ for temperature fluctuations between positions 2 and 5 (red curve) and for image intensity variations between positions 7 and 8 (blue curve). The measurements are made at $\mathrm{Ra}=4.6 \times 10^{9}$. 
$A, B$

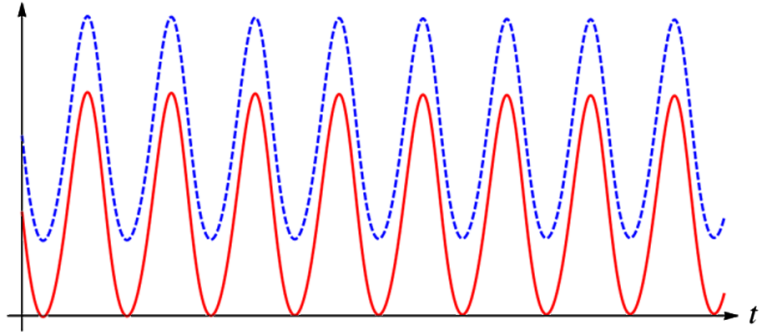

FIG. 6 (color online). Numerical solution of system (1) for $A(t)$ (red solid line) and $B(t)$ (blue dashed line) with $r=\mu=1$, $c=0.5$, and $r \tau_{0}=1.08(\pi / 2)$ (just above the threshold). For clarity, $B(t)$ has been shifted by a constant.

equations, describing the amplitudes $A(t)$ and $B(t)$ of the instabilities in the two boundary layers, respectively,

$\dot{A}(t)=r A(t)-\mu A(t)\left[A\left(t-\tau_{0}\right)+c B\left(t-\tau_{0}\right)\right]$,

$\dot{B}(t)=r B(t)-\mu B(t)\left[B\left(t-\tau_{0}\right)+c A\left(t-\tau_{0}\right)\right]$.

Here $r$ represents a growth rate of the boundary layer instabilities, $\mu$ is a parameter to set the oscillation amplitude, and $c(0<c<1)$ is a coupling constant having a typical value of 0.5 . The delay time $\tau_{0}$ reflects the time interval for disturbances (thermal plumes) to travel from one boundary layer to the other. System (1) is written in a slightly different form from that used in Ref. [12] (by using the absolute value rather than the square of the amplitude in the delayed saturation terms), but it maintains all the essential features of the original model.

System (1) presents a fixed point for $A=B=(r / \mu) \times$ $[1 /(1+c)]$. Linear stability reveals that system (1) destabilizes as soon as $r \tau_{0}>\pi / 2$, leading to an in-phase oscillatory solution for $A(t)$ and $B(t)$ with weak modulations around the fixed point close to threshold, as seen in Fig. 6. The corresponding oscillation frequency $f_{0}$ is such that $2 \pi f_{0}=r$, leading to $f_{0} \tau_{0}=1 / 4$ in that limit. Away from the threshold, i.e., when $r \tau_{0} \gg \pi / 2$, the system bifurcates to a strongly nonlinear regime, in which $A(t)$ and $B(t)$ oscillate out of phase, as shown in Ref. [12]. In this case, the growth rate of both $A(t)$ and $B(t)$ is maximized when the amplitude of the companion oscillator is close to zero, hence the phase opposition.

From the shadowgraph movie in Ref. [16], we find that most warm plumes erupt from the lower one-half of the conducting plate between positions 3 and 7 . The mixing of the incoming cold plumes takes place in the other half of the lower conducting plate. Therefore, the average traveling distance for the warm plumes to trigger the instabilities in the upper boundary layer is from positions 6 to 1 . The corresponding traveling time is $\tau_{0} \simeq \pi D /\left(4 U_{3}\right)$. From the measured $f_{0} \tau_{0}$ in Fig. 3 , we find that the condition $f_{0} \tau_{0}=1 / 4$ set by the marginal stability condition of system (1) is indeed satisfied in the experiment.
Our findings thus demonstrate the existence of a direct coupling between the top and bottom boundary layers, which is mediated by LSC and is responsible for a global oscillation, in the absence of other possible mechanisms. Both the in-phase oscillation between the two boundary layers and the quantitative comparison of its frequency in Fig. 3 support this scenario. Moreover, they also suggest that the convective flow in this cell is at a critical state, near the global instability threshold [in the sense of system (1)]. We notice that this is a special state under the predicted conditions, which may not be satisfied in other cell geometries $[9,13,18]$. In fact, we have found that, in a long horizontal cell of aspect ratio unity, the oscillations observed in the total heat flux disappeared [17]. We suspect that this is a "dephasing" process associated with an increased amount of incoherent emission of thermal plumes over a larger area of the conducting plate, whose intrinsic conductivity may also matter.

This work was supported by RGC of Hong Kong SAR under Grant No. HKUST603208.

[1] G. Ahlers et al., Rev. Mod. Phys. 81, 503 (2009).

[2] D. Lohse et al., Annu. Rev. Fluid Mech. 42, 335 (2010).

[3] B. Castaing et al., J. Fluid Mech. 204, 1 (1989).

[4] M. Sano, X.Z. Wu, and A. Libchaber, Phys. Rev. A 40, 6421 (1989).

[5] X.-L. Qiu, S. H. Yao, and P. Tong, Phys. Rev. E 61, R6075 (2000).

[6] X.-L. Qiu and P. Tong, Phys. Rev. Lett. 87, 094501 (2001); Phys. Rev. E 66, 026308 (2002).

[7] D. Funfschilling and G. Ahlers, Phys. Rev. Lett. 92, 194502 (2004).

[8] C. Resagk et al., Phys. Fluids 18, 095105 (2006).

[9] H.-D. Xi et al., Phys. Rev. Lett. 102, 044503 (2009).

[10] S. Cioni et al., J. Fluid Mech. 335, 111 (1997); J.J. Niemela et al., J. Fluid Mech. 449, 169 (2001); R. du Puits, C. Resagk, and A. Thess, Phys. Rev. E 75, 016302 (2007); S.-Q. Zhou, C. Sun, and K. Q. Xia, Phys. Rev. E 76, 036301 (2007).

[11] L. N. Howard, in Proceedings of the 11th International Congress of Applied Mechanics, edited by Henry Goertler (Springer, Berlin, 1966), p. 1109.

[12] E. Villermaux, Phys. Rev. Lett. 75, 4618 (1995).

[13] E. Brown and G. Ahlers, Phys. Fluids 20, 105105 (2008); J. Fluid Mech. 638, 383 (2009).

[14] F. F. Araujo, S. Grossmann, and D. Lohse, Phys. Rev. Lett. 95, 084502 (2005); K. Sugiyama et al., Phys. Rev. Lett. 105, 034503 (2010).

[15] H. Song and P. Tong, Europhys. Lett. 90, 44001 (2010).

[16] The large-scale flow is more visible in the movie at http:// physics.ust.hk/penger/LSC.htm.

[17] H. Song, Ph.D. thesis, HKUST, 2011.

[18] R. du Puits et al. (unpublished). 